UPRF 95-426

LPTHE-95-40

July 1995

\title{
Spontaneous symmetry breaking with Wilson renormalization group
}

\author{
M. Bonini \\ Dipartimento di Fisica, Università di Parma and \\ INFN, Gruppo Collegato di Parma, Italy \\ and \\ M. D'Attanasio \\ Laboratoire de Physique Théorique et Hautes Energies, Université Pierre et Marie Curie \\ (Paris VI) et Université Denis Diderot (Paris VII), Tour 16, 1er. étage, 4, Place Jussieu \\ 75252 Paris, Cedex 05, France. Laboratoire Associé au CNRS URA280 and \\ INFN, Gruppo Collegato di Parma, Italy
}

\begin{abstract}
We study the conditions under which a symmetry is spontaneously broken in the Wilson renormalization group formulation. Both for a global and local symmetry, the result is that in perturbation theory one has to perform a fine tuning of the boundary conditions for the flow of the relevant couplings. We consider in detail the discrete $Z_{2}$ case and the Abelian Higgs model.
\end{abstract}

${ }^{*}$ Della Riccia fellow 


\section{Introduction}

One might think that theories in which one has to deal with an infinite number of interactions are not predictive. On the other hand in many cases only a finite number of these couplings are independent while the others can be expressed in terms of the independent ones. This is a general feature whenever one considers the effective theory of a more fundamental theory in which there are symmetries which are apparently broken at the effective level, that is at low energies. A similar situation happens when a (non-anomalous) symmetry of a theory is broken at the quantum level by the regularization as for instance in the case of chiral gauge theories [1]. In general if there is an infinite number of couplings one can derive [2] a set of conditions which constrain them, preserving renormalizability. One studies the dependence of the couplings on the ultraviolet cutoff and seeks solutions of the renormalization group equations in which only a finite number of couplings are allowed to be independent function of the cutoff with the constrain that all remaining interactions vanish when the independent ones are zero. It is conjectured that the solutions of these conditions are the values of the couplings which restore some symmetry of the theory, hidden by the presence of an infinity number of interactions. Then it is also conjectured that in perturbation theory it is sufficient to restore symmetries at one value of the cutoff after which the symmetry will be automatically maintained. This problem of reducing the number of coupling can be analysed in the Wilson renormalization group ( $R G)$ [3]-[6]. In this formulation one introduces a Wilsonian effective action at a scale $\Lambda$ in order to take into account the modes above $\Lambda$. The invariance of the physical Green functions with respect to variations of $\Lambda$, gives a flow equation for this effective action.

Recently the RG method has been extended to gauge theories [6]-[9]. In this case the various couplings do not flow independently. In particular once the relevant couplings (i.e. renormalizable interactions) are fine tuned at some scale, the evolution of the Wilsonian action is constrained by the symmetry. For instance in [6, 7] it is shown that for the $\mathrm{SU}(2)$ Yang-Mills theory there are 9 relevant couplings which are all expressed in terms of the vector three point coupling $g$ at some scale (after having fixed the normalization of the fields) by solving the so called "fine-tuning" equations. Then one prove that the BRS invariance of the physical effective action is recovered.

A small number of independent couplings is a characteristic feature also in the case in which the symmetry is spontaneously broken. In this case the symmetry is broken by the vacuum. In perturbation theory one does not find terms which break the symmetry so they must be introduced by hand as interactions and only for a specific value of their couplings one recovers spontaneous symmetry breaking [2].

In this paper we consider the problem of the implementation of a spontaneously broken symmetry in the RG framework. One has to distinguish the global symmetry case from the local one. At first sight the latter is more complicated since the introduction of a cutoff breaks the symmetry itself. However due to the Ward identities associated to the local symmetry this case can be analyzed as the symmetric one. One introduces the operator which gives the breaking of the Ward identities due to the presence of the scale $\Lambda$ and studies its flow with $\Lambda$. The boundary conditions for the relevant part of this operator determine if one deals with a symmetry which is spontaneously broken or not. Having 
chosen one of the two implementations the flow constraints the couplings in such a way that the symmetry (spontaneously broken or not) is maintained for any value of $\Lambda$.

The signature of a spontaneously broken symmetry is the fact that one scalar field $\phi_{0}$ acquires a non-vanishing vacuum expectation value (vev) $\left\langle\phi_{0}\right\rangle$. Thus the theory is symmetric in the unphysical field $\phi_{0}$ but it is not in the physical field $\phi=\phi_{0}-\left\langle\phi_{0}\right\rangle$. In this paper we study this property at the effective level. Namely if the theory is symmetric at any $\Lambda$ in some unphysical field $\phi_{0}=\phi+v$. We find in general that in perturbation theory $v$ is $\Lambda$-independent but gets loop corrections. Besides the field $\phi$ (and then also $\left.\phi_{0}=\phi+v\right)$ has a $\Lambda$-dependent value of the vev, which vanishes only for $\Lambda=0$. In many cases this running of $\langle\phi\rangle$ gives an important contribution to the running of the masses of the particles. For instance in [10] it was shown how the running of the vev of the dilaton may be of help in solving the problem of unitarity in some gauge theories of gravity.

After a brief description of the RG method given in section 2, we consider in section 3 the case of a scalar theory with a spontaneously broken $Z_{2}$ symmetry. As an example of local symmetries, in section 4 we analyze in detail the Abelian Higgs model. Section 5 contains some conclusions.

\section{Wilson effective action}

In this section we recall the main features of the Wilson approach for a simple Euclidean theory with one scalar field, in order to simplify the notation. The results can be generalized easily to more realistic theories.

The generating functional is

$$
Z[j]=e^{-W[j]}=\int \mathcal{D} \phi \exp \left\{-\frac{1}{2}\left(\phi, D^{-1} \phi\right)_{0 \Lambda_{0}}+(j, \phi)_{0 \Lambda_{0}}-S_{\text {int }}\left[\phi ; \Lambda_{0}\right]\right\},
$$

where $D$ is the free propagator of the theory. We have introduced a cutoff scalar product

$$
(A, B)_{\Lambda \Lambda_{0}} \equiv \int_{p} K_{\Lambda \Lambda_{0}}^{-1}(p) A(-p) B(p), \quad \int_{p} \equiv \int \frac{d^{4} p}{(2 \pi)^{4}}
$$

where $K_{\Lambda \Lambda_{0}}(p)$ is a cutoff function which is one for $\Lambda^{2} \leq p^{2} \leq \Lambda_{0}^{2}$ and rapidly vanishing outside this interval. $S_{\text {int }}\left[\phi ; \Lambda_{0}\right]$ is the UV action involving monomials in the fields and their derivatives which have dimension not larger than four and are Lorentz scalars. The Wilsonian effective action $S_{\text {eff }}$ is defined by integrating over the energy modes higher than $\Lambda$. One finds

$$
Z[j]=N\left[j ; \Lambda, \Lambda_{0}\right] \int \mathcal{D} \phi \exp \left\{-\frac{1}{2}\left(\phi, D^{-1} \phi\right)_{0 \Lambda}+(j, \phi)_{0 \Lambda}-S_{\mathrm{eff}}\left[\phi ; \Lambda, \Lambda_{0}\right]\right\}
$$

where the coefficient $N$ is given by

$$
\log N\left[j ; \Lambda, \Lambda_{0}\right]=\frac{1}{2}(j, D j)_{0 \Lambda_{0}}-\frac{1}{2}(j, D j)_{0 \Lambda} .
$$




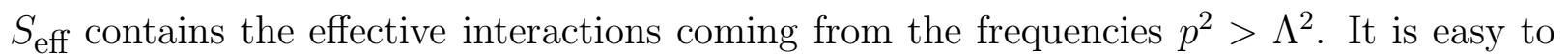
show how this functional is equivalent to a generalization of (1), in which the free propagator contains $\Lambda$ as an infrared cutoff. More precisely we have

$$
S_{\mathrm{eff}}\left[\phi ; \Lambda, \Lambda_{0}\right]-\frac{1}{2}\left(\phi, D^{-1} \phi\right)_{\Lambda \Lambda_{0}} \equiv W\left[D^{-1} K_{\Lambda \Lambda_{0}}^{-1}(p) \phi ; \Lambda, \Lambda_{0}\right]
$$

where

$$
e^{-W\left[j ; \Lambda, \Lambda_{0}\right]}=\int \mathcal{D} \phi \exp \left\{-\frac{1}{2}\left(\phi, D^{-1} \phi\right)_{\Lambda_{\Lambda}}+(j, \phi)_{0 \Lambda_{0}}-S_{\text {int }}\left[\phi ; \Lambda_{0}\right]\right\} .
$$

Namely, apart from the tree level two-point function, the Wilsonian effective action is the generating functional of the connected Green functions with an IR cutoff $\Lambda$ and amputated of the free external propagators. As one expects, it is technically easier to study the Legendre transform of $W\left[j ; \Lambda, \Lambda_{0}\right]$, which is usually called "cutoff effective action" and is a generalization of the usual quantum effective action, since it contains the infrared cutoff $\Lambda$ in the free propagators. In the limit $\Lambda \rightarrow 0$ and $\Lambda_{0} \rightarrow \infty$, one recovers the physical quantum effective action. Both these limits can be taken in perturbation theory. In particular the dependence on the ultraviolet cutoff $\Lambda_{0}$ will be often understood.

From the fact that the $\Lambda$-dependence of the cutoff effective action $\Gamma\left[\phi ; \Lambda, \Lambda_{0}\right]$ is only coming from the free propagators, one finds a flow equation in $\Lambda$, the exact RG equation, which in general has the form

$$
\Lambda \partial_{\Lambda} \Gamma\left[\phi ; \Lambda, \Lambda_{0}\right]=I\left[\Gamma ; \Lambda, \Lambda_{0}\right]
$$

where $I$ depends non-linearly on $\Gamma$. For the precise form of $I$ in the various cases see [11, 12]. In order to integrate equation (3) one has to supply the boundary conditions. For this reason it is useful to split the cutoff effective action into two parts. One performs a Taylor expansion of the cutoff vertices at vanishing momenta (if there are massless particles the expansion should be done around a non-vanishing subtraction point). This expansion will have coefficients of decreasing dimension. These coefficients are the couplings of the theory. The "relevant" part is obtained by keeping the terms with coefficients having nonnegative dimension (relevant couplings). The remaining part is called "irrelevant". For instance in the scalar case one gets for the relevant part

$$
\Gamma_{\text {rel }}[\phi ; \Lambda]=\frac{1}{2} \int d^{4} x \phi(x)\left[\sigma_{1}(\Lambda)-\sigma_{2}(\Lambda) \partial^{2}\right] \phi(x)+\frac{\sigma_{3}(\Lambda)}{4 !} \int d^{4} x \phi^{4}(x) .
$$

Since we expect the theory to be renormalizable, for $\Lambda \sim \Lambda_{0}$ the dimension of the irrelevant couplings should be given only by powers of $\Lambda_{0}$. Thus the simplest boundary condition for the irrelevant part of the cutoff effective action is

$$
\Gamma_{\operatorname{irr}}\left[\phi ; \Lambda=\Lambda_{0}\right]=0
$$

However it would not give any problem to consider irrelevant couplings which vanish only in the limit $\Lambda_{0} \rightarrow \infty$.

The boundary conditions for the relevant part fix the physical couplings. Thus it is natural to set them at the physical point $\Lambda=0$. For the scalar case this requirement fixes all the three parameters to be $\sigma_{1}(\Lambda=0)=m^{2}, \sigma_{2}(\Lambda=0)=1$ and $\sigma_{3}(\Lambda=0)=g$. In the usual field theory language this corresponds to give the renormalization conditions. For a 
more realistic theory the number of relevant couplings is larger than the physical conditions. This is related to the fact that some couplings are constrained by the symmetries of the theory. In general the implementation of a symmetry is equivalent to a certain number of "fine tuning" conditions which fix the undetermined couplings in terms of the physical ones. For instance in the SU(2) Yang-Mills case one has 9 parameters, of which 3 are physical conditions (the vector and ghost wave function normalization and the gauge coupling) and the remaining are given by 6 fine tuning conditions [6, 7].

Once the boundary conditions are fixed, the cutoff effective action can be obtained by integrating the RG equation

$$
\Gamma[\phi ; \Lambda]=\Gamma_{\mathrm{rel}}[\phi ; \Lambda=0]+\int_{0}^{\Lambda} \frac{d \lambda}{\lambda} I_{\mathrm{rel}}+\int_{\Lambda}^{\Lambda_{0}} \frac{d \lambda}{\lambda} I_{\mathrm{irr}},
$$

where the first term provides the boundary conditions for the relevant part, i.e. the physical conditions. The iterative solution of this integral equation gives the renormalized loop expansion in terms of the physical couplings [4, 6, 11].

\section{Scalar case}

We want to describe the case of a $Z_{2}$ invariant scalar field $\phi_{0}$ with a tree level negative mass parameter $\mu^{2}$. This means that $\phi_{0}$ is not the physical field of the theory and $\mu^{2}$ is not the mass of the scalar particle we are describing. The effective action expressed in terms of the physical field $\phi$ has no longer any $Z_{2}$ symmetry. However we impose that the effective action depends on the field $\phi$ only through the combination $(\phi+v)^{2}$, where $v$ is some suitable momentum independent and $\Lambda$-independent quantity, corresponding to the vacuum expectation value (vev) of the "unphysical" field $\phi_{0}$.

The choice of the boundary conditions for the relevant part of the cutoff effective action

$\Gamma_{\mathrm{rel}}[\phi ; \Lambda]=\int d^{4} x\left\{\rho(\Lambda) \phi(x)+\frac{1}{2} \phi(x)\left[\sigma(\Lambda)-z(\Lambda) \partial^{2}\right] \phi(x)+\frac{1}{3 !} g_{3}(\Lambda) \phi^{3}(x)+\frac{1}{4 !} g(\Lambda) \phi^{4}(x)\right\}$

must be such that we fix the physical couplings (the mass $m$ and four-point coupling $g$ ) and the residual "reflection" symmetry corresponding to the spontaneously broken $Z_{2}$ symmetry. As expected, this will fix both the value of $g_{3}=g_{3}(\Lambda=0)$ and the vev $v$, in terms of $m$ and $g$. Indeed, as we shall see, the parameter $v$ is the vev of the field $\phi_{0}$ only at $\Lambda=0$, while in general $\left\langle\phi_{0}\right\rangle$ is $\Lambda$-dependent. So for the moment we write

$$
\Gamma_{\text {rel }}[\phi ; \Lambda=0]=\int d^{4} x\left\{\frac{1}{2} \phi(x)\left(-\partial^{2}+m^{2}\right) \phi(x)+\frac{1}{3 !} g_{3} \phi^{3}(x)+\frac{1}{4 !} g \phi^{4}(x)\right\} .
$$

Now we come to the determination of $g_{3}$ and $v$. It is convenient to consider the cutoff effective potential $V$

$$
V(\phi ; \Lambda)=\sum_{n=1}^{\infty} \frac{1}{n !} \Gamma_{n}(\Lambda) \phi^{n}, \quad \Gamma_{n}(\Lambda)=\left.\Gamma_{n}\left(p_{1}, \ldots, p_{n} ; \Lambda\right)\right|_{p_{i}=0} .
$$


Notice that also $V$ can be split into its relevant part $V_{\text {rel }}=\sum_{n=1}^{4} \frac{1}{n !} \Gamma_{n}(\Lambda) \phi^{n}$ and irrelevant part $V_{\text {irr }}=\sum_{n=5}^{\infty} \frac{1}{n !} \Gamma_{n}(\Lambda) \phi^{n}$. The fact that $V$ is depending on $(\phi+v)^{2}$, means that $V(\phi-$ $v ; \Lambda)$ is even in $\phi$. Then, by writing

$$
V(\phi-v ; \Lambda)=\sum_{n=1}^{\infty} \frac{1}{n !} G_{n}(\Lambda) \phi^{n},
$$

where

$$
G_{n}(\Lambda)=\sum_{k=n}^{\infty} \frac{1}{(k-n) !} \Gamma_{k}(\Lambda)(-v)^{k-n},
$$

we get the relations

$$
G_{2 n+1}=0 .
$$

Notice that the functions $G_{n}$ are the zero momentum vertices of the unbroken theory and it holds the relation

$$
G_{n}(\Lambda)=\left.\frac{\partial^{n} V(\phi ; \Lambda)}{\partial \phi^{n}}\right|_{\phi=-v} .
$$

We will use the two equations $G_{1}=0$ and $G_{3}=0$, namely

$$
V^{\prime}(-v ; \Lambda)=0, \quad V^{\prime \prime \prime}(-v ; \Lambda)=0,
$$

at $\Lambda=0$ to compute the boundary condition $g_{3}$ and the vev $v$. In the following we will consider eq. (6) in perturbation theory. At the tree level one finds

$$
v^{(0)}=\sqrt{\frac{3 m^{2}}{g}}, \quad g_{3}^{(0)}=\sqrt{3 m^{2} g},
$$

independent of $\Lambda$ and satisfying $g_{3}^{(0)}=g v^{(0)}$. At one loop order (6) gives for the boundary condition of the three point vertex

$$
g_{3}^{(1)}=-\frac{g}{2 m^{2}} V_{\mathrm{irr}}^{\prime(1)}\left(-v^{(0)} ; \Lambda=0\right)-\frac{1}{4} V_{\mathrm{irr}}^{\prime \prime \prime}(1)\left(-v^{(0)} ; \Lambda=0\right)
$$

and for the vev

$$
v^{(1)}=-\frac{1}{2 m^{2}} V_{\mathrm{irr}}^{\prime(1)}\left(-v^{(0)} ; \Lambda=0\right)+\frac{3}{4 g} V_{\mathrm{irr}}^{\prime \prime \prime}{ }^{(1)}\left(-v^{(0)} ; \Lambda=0\right) .
$$

Notice that equation (7) gives $g_{3}$ in terms of irrelevant vertices of the effective action evaluated at zero momenta. This is a general feature and allows one to deduce the perturbative expansion since in the iterative solution of the RG equations irrelevant vertices al loop $\ell$ involve relevant couplings at lower loops $\ell^{\prime}<\ell$. From the irrelevant part of the effective potential at one loop (see appendix A) one finds

$$
g_{3}^{(1)}=-\frac{3 m g \sqrt{3 g}}{64 \pi^{2}}, \quad v^{(1)}=\frac{9 m \sqrt{3 g}}{64 \pi^{2}} .
$$

With this boundary condition for the three point coupling the one-loop effective potential is completely determined and it is given by

$$
64 \pi^{2} V^{(1)}[\phi ; \Lambda]=-\sqrt{3 g} m^{3} \phi-5 g m^{2} \phi^{2}-3 m g \sqrt{3 g} \phi^{3}-\frac{9 g^{2}}{8} \phi^{4}-\frac{g}{2} \Lambda^{2}\left(\phi+v^{(0)}\right)^{2}
$$




$$
+\left[\left(\frac{g}{2}\left(\phi+v^{(0)}\right)^{2}-\frac{m^{2}}{2}\right)^{2}-\Lambda^{4}\right] \log \left(\frac{\Lambda^{2}+\frac{g}{2}\left(\phi+v^{(0)}\right)^{2}-\frac{m^{2}}{2}}{m^{2}}\right) .
$$

Notice that the one point function vanishes only at $\Lambda=0$ and is quadratically divergent for large $\Lambda$. This implies that at any non-vanishing $\Lambda$ the value $\phi=0$ is not the minimum of the cutoff effective potential. The running minimum is defined by

$$
\left.\frac{\partial V(\phi ; \Lambda)}{\partial \phi}\right|_{\phi=v(\Lambda)}=0
$$

and at one loop it is given by

$$
v(\Lambda)=-\frac{1}{m^{2}} \rho(\Lambda)=\frac{\sqrt{3 g} m}{32 \pi^{2}}\left[-\frac{\Lambda^{2}}{m^{2}}+\log \left(\frac{\Lambda^{2}+m^{2}}{m^{2}}\right)\right] .
$$

Thus we have that the running of the one loop vacuum expectation value of the unphysical field $\phi_{0}$ is given by

$$
\left\langle\phi_{0}\right\rangle=v^{(1)}-\frac{1}{m^{2}} \rho(\Lambda)=\frac{m \sqrt{3 g}}{32 \pi^{2}}\left[\frac{\Lambda^{2}}{m^{2}}+\frac{9}{2}-\log \left(\frac{\Lambda^{2}+m^{2}}{m^{2}}\right)\right] .
$$

As well known the loop expansion is insensitive to translations of the field [13]. This implies that at any loop $\ell$ the contribution to $V^{(\ell)}$ coming from the graphs is the same in the broken and unbroken theory, namely is a function of $\phi_{0}^{2}=\left(\phi+v^{(0)}\right)^{2}$. Thus the graphs contribution in any odd derivative of $V^{(\ell)}$ evaluated at $\phi=-v^{(0)}$ is vanishing. The only thing which remains in $\left.\frac{\partial^{2 n+1}}{\partial \phi^{2 n+1}} V^{(\ell)}\right|_{\phi=-v^{(0)}}$ is the contribution from the boundary conditions, which is independent of $\Lambda$. This argument implies that $V^{(1)}\left(-v^{(0)} ; \Lambda\right)$ and $V^{\prime \prime \prime}(1)\left(-v^{(0)} ; \Lambda\right)$ are independent of $\Lambda$, giving a $\Lambda$-independent one loop correction to the vev. This observation holds at any loop order. At two loops for instance we have from (6)

$$
\begin{aligned}
& V^{\prime(0)}\left(-v^{(2)} ; \Lambda\right)+V^{\prime(1)}\left(-v^{(1)} ; \Lambda\right)+V^{\prime(2)}\left(-v^{(0)} ; \Lambda\right)=0, \\
& V^{\prime \prime \prime}(0)\left(-v^{(2)} ; \Lambda\right)+V^{\prime \prime \prime}(1)\left(-v^{(1)} ; \Lambda\right)+V^{\prime \prime \prime}(2)\left(-v^{(0)} ; \Lambda\right)=0,
\end{aligned}
$$

where, for the same reason explained above, $V^{(1)}$ is even in $\phi+v^{(0)}+v^{(1)}$ and $V^{(2)}$ is even in $\phi+v^{(0)}$, apart from the boundary conditions. This justifies in perturbation theory our assumption of a $\Lambda$-independent value of $v$.

Let us conclude this section with some comments about massless theories. Because of infrared divergences, some relevant couplings (more precisely the marginal ones) are defined as the value of the corresponding vertices at some non-vanishing subtraction points. At $\Lambda=0$ the various vertices at zero momenta are divergent but the effective potential is well defined since the scale $\bar{\mu}$ defining the subtraction points acts as a mass term in the loop integrals. In massless theories there is the possibility that spontaneous symmetry breaking is driven by radiative corrections. This corresponds to having a vanishing tree level value for $g_{3}$ and $v$. It is immediate to see that (6) implies that $g_{3}$ and $v$ vanish at any loop order. Thus it impossible to have perturbative spontaneous symmetry breaking for a massless scalar theory. This corresponds to the observation made by Coleman and Weinberg [13] that the non-trivial minimum of the effective potential is out of the validity 
of the perturbative expansion. However, there is a possibility to circumvent this difficulty. Imagine that the boundary condition for the coupling $g(\Lambda)$ gets loop corrections

$$
g(\Lambda=0)=g+a \not h g^{2},
$$

where $a$ is some coefficient. It is immediate to check that in this case the set of equation (6) admits a solution for the tree level quantities $g_{3}^{(0)}$ and $v^{(0)}$, which clearly depends on the scale used for the subtraction point. A boundary condition like (12) is somewhat "unphysical", since now $g$ is no more the value of the physical four point coupling, and has no clear meaning. On the other hand if we are dealing with a theory with two or more fields, in principle it is possible to give a boundary condition for the self-interaction coupling of one field in a manner analogous to (12), namely as a series in another coupling, defined through the second field. This is precisely what was done by Coleman and Weinberg for the massless scalar QED, in which the four-point coupling of the scalar is fixed to be zero at tree level and proportional (with a precise factor) to $e^{4}$ at one loop, so that a non-vanishing tree level vev $v$ is obtained 1 . This is an example of how the request of symmetry brings us to make a "fine tuning" of one or more boundary conditions.

\section{Abelian gauge symmetry}

In this section we consider the abelian Higgs model in four dimensional Euclidean space with spontaneously broken $U(1)$ gauge symmetry. The "classical action" for this model is

$$
S=\int d^{4} x\left\{\frac{1}{4} F_{\mu \nu} F_{\mu \nu}+\left|D_{\mu} \phi\right|^{2}-\frac{1}{2} m^{2}|\phi|^{2}+g|\phi|^{4}+\frac{1}{2 \alpha}\left(\partial A-\alpha M \phi_{2}\right)^{2}+\bar{c}\left(\partial^{2}-\alpha M^{2}-\alpha e M \phi_{1}\right) c\right\},
$$

where $D_{\mu}=\partial_{\mu}-i e A_{\mu}, \phi=\frac{1}{\sqrt{2}}\left(\phi_{1}+\frac{M}{e}+i \phi_{2}\right)$, the masses and couplings are in the relation $2 g M^{2}=e^{2} m^{2}$ and we have included the 't Hooft gauge fixing term and the ghost action. This action can be rewritten as

$$
\begin{gathered}
S=\int d^{4} x\left\{\frac{1}{4} F_{\mu \nu} F_{\mu \nu}+\frac{1}{2} M^{2} A^{2}+\frac{1}{2 \alpha}(\partial A)^{2}+\frac{1}{2}\left|D_{\mu} \phi_{1}\right|^{2}+\frac{1}{2} m^{2} \phi_{1}^{2}\right. \\
+\frac{1}{2}\left|D_{\mu} \phi_{2}\right|^{2}+\frac{1}{2} \alpha M^{2} \phi_{2}^{2}+\bar{c}\left(\partial^{2}-\alpha M^{2}\right) c \\
\left.+\frac{g}{4}\left(\phi_{1}^{4}+\phi_{2}^{4}+2 \phi_{1}^{2} \phi_{2}^{2}+4 \frac{M}{e} \phi_{1} \phi_{2}^{2}+4 \frac{M}{e} \phi_{1}^{3}\right)+e A_{\mu}\left(\phi_{2} \partial_{\mu} \phi_{1}-\phi_{1} \partial_{\mu} \phi_{2}\right)+e M A^{2} \phi_{1}-\alpha e M \bar{c} \phi_{1} c\right\} .
\end{gathered}
$$

In this form it is easy to see that the free propagators are

$$
\begin{gathered}
D_{\mu \nu}(p)=\frac{1}{p^{2}+M^{2}}\left(\delta_{\mu \nu}-\frac{1-\alpha}{p^{2}+\alpha M^{2}} p_{\mu} p_{\nu}\right), \quad D_{\bar{c} c}(p)=\frac{-1}{p^{2}+\alpha M^{2}}, \\
D_{1}=\frac{1}{p^{2}+m^{2}}, \quad D_{2}=\frac{1}{p^{2}+\alpha M^{2}} .
\end{gathered}
$$

The action (13) is invariant under the BRS transformations 15, 16]

$$
\delta A_{\mu}=\eta \partial_{\mu} \bar{c}, \quad \delta \bar{c}=0, \quad \delta c=\eta \frac{1}{\alpha}\left(\partial A-\alpha M \phi_{2}\right),
$$

\footnotetext{
${ }^{1} \mathrm{~A}$ numerical study of a truncation of RG equations for massless scalar QED was performed in [14].
} 


$$
\delta \phi_{1}=-\eta e \phi_{2} \bar{c}, \quad \delta \phi_{2}=\eta\left(M \bar{c}+e \phi_{1} \bar{c}\right),
$$

where $\eta$ is a Grassmann parameter. This invariance is expressed by a set of Slavnov-Taylor $(\mathrm{ST})$ identities $\mathcal{S} W=0$ for the generating functional $W[J, \chi]$ of the quantum correlation functions

$$
e^{-W[J, \chi]}=\int \mathcal{D} \phi e^{-S+S_{J}+S_{\chi}},
$$

where the source terms are $S_{J}=\int d^{4} x\left[j_{\mu} A_{\mu}+j_{1} \phi_{1}+j_{2} \phi_{2}+\bar{j} c+\bar{c} j\right]$ and $S_{\chi}=\int d^{4} x\left[-\chi_{1} e \phi_{2} \bar{c}+\right.$ $\left.\chi_{2}\left(M \bar{c}+e \phi_{1} \bar{c}\right)\right]$. The $\mathrm{ST}$ operator is

$$
\mathcal{S}=\left(\partial_{\mu} j_{\mu}\right) \frac{\delta}{\delta j}+M \bar{j} \frac{\delta}{\delta j_{2}}-\frac{1}{\alpha} \bar{j} \partial_{\mu} \frac{\delta}{\delta j_{\mu}}+j_{1} \frac{\delta}{\delta \chi_{1}}+j_{2} \frac{\delta}{\delta \chi_{2}} .
$$

For the quantum effective action, defined as $\Gamma[\Phi, \chi]=W[J, \chi]+S_{J}$, the ST identities are $\mathcal{S}_{\Gamma} \Gamma=0$, where the Slavnov operator is

$$
\mathcal{S}_{\Gamma}=\left(\partial_{\mu} \bar{c}\right) \frac{\delta}{\delta A_{\mu}}+\frac{1}{\alpha}\left(\partial_{\mu} A_{\mu}-\alpha M \phi_{2}\right) \frac{\delta}{\delta c}-\frac{1}{2} \sum_{i=1,2}\left(\frac{\delta \Gamma}{\delta \phi_{i}} \frac{\delta}{\delta \chi_{i}}+\frac{\delta \Gamma}{\delta \chi_{i}} \frac{\delta}{\delta \phi_{i}}\right) .
$$

These expressions can be simplified by noting that the identity $\bar{j}+\partial^{2} \frac{\delta W}{\delta j}+\alpha M \frac{\delta W}{\delta \chi_{2}}=0$ holds. In terms of $\Gamma$ it reads

$$
\frac{\delta \Gamma}{\delta c}+\partial^{2} \bar{c}+\alpha M \frac{\delta \Gamma}{\delta \chi_{2}}=0
$$

Using this relation the ST identities become

$$
\bar{c} \partial_{\mu} \frac{\delta \Gamma^{\prime}}{\delta A_{\mu}}+\sum_{i=1,2} \frac{\delta \Gamma^{\prime}}{\delta \phi_{i}} \frac{\delta \Gamma^{\prime}}{\delta \chi_{i}}=0
$$

where $\Gamma^{\prime}=\Gamma-\frac{1}{2 \alpha}\left(\partial_{\mu} A_{\mu}-\alpha M \phi_{2}\right)^{2}$. The relevant part of $\Gamma$ contains 22 couplings corresponding to the renormalizable interactions (see appendix $\mathrm{B}$ ), whose values are fixed by the renormalization conditions. We will see that 6 of them are given by physical requirements (masses and wave function normalizations of $A_{\mu}$ and $\phi_{1}$, the electric charge $e$ and the vanishing of the $\phi_{2}-A$ mixing in the two point function) while the others will be constrained by the symmetry.

We now apply the Wilson method. By integrating in the path integral the higher momentum modes $\left(p^{2}>\Lambda^{2}\right)$ we get

$$
e^{-W[J, \chi]}=N \int \mathcal{D} \phi \exp \left\{\left(-S_{2}+S_{J}+\chi_{2} M \bar{c}\right)_{0 \Lambda}-S_{\text {eff }}\right\}
$$

where $S_{2}$ is the part of the action (13) quadratic in the fields and the notation $(\cdots)_{0 \Lambda}$ stands for the cutoff scalar product introduced in section 2. The factor $N$ in (15) is given by the product of the four terms obtained from (2) with the substitutions $j \rightarrow\left\{j_{\mu}, j_{1}, j_{2},-\bar{j}\right\}$ and $D j \rightarrow\left\{D_{\mu \nu} j_{\nu}, D_{1} j_{1}, D_{2} j_{2}, D_{\bar{c} c}\left(j-M \chi_{2}\right)\right\}$. The functional $S_{\text {eff }}[\Phi, \chi ; \Lambda]$ is the Wilsonian effective action and is given by

$$
\exp \left\{-\left(S_{2}\right)_{\Lambda \Lambda_{0}}-S_{\text {eff }}[\Phi, \chi ; \Lambda]\right\}=\int \mathcal{D} \phi \exp \left\{\left(-S_{2}+\chi_{2} M \bar{c}\right)_{\Lambda \Lambda_{0}}-S_{\text {int }}+S_{J}\right\}
$$


where the sources in the r.h.s. are related to the field $\Phi$ by

$$
\Phi=K_{\Lambda \Lambda_{0}}\left(D_{\mu \nu} j_{\nu}, D_{1} j_{1}, D_{2} j_{2},-D_{\bar{c} c} \bar{j},-D_{\bar{c} c} j+K_{\Lambda \Lambda_{0}}^{-1} D_{\bar{c} c} M \chi_{2}\right)
$$

and $S_{\text {int }}$ is the UV action involving monomials in the fields, BRS sources and their derivatives which have dimension not higher than four, are Lorentz scalar and are invariant under charge conjugation. This, together with the high momentum behaviour of the free propagators (14) ensures perturbative renormalizability [11.

In the RG formulation one has to define the boundary conditions for the flow in $\Lambda$ of the Wilsonian action $S_{\text {eff }}$. As usual its irrelevant part is fixed at $\Lambda=\Lambda_{0}$ and vanishes for $\Lambda_{0} \rightarrow \infty$. The relevant part of $S_{\text {eff }}$ is fixed at the physical point $\Lambda=0$ in such a way that the the physical effective action fulfills the ST identities. In order to show how to fix the parameters of this functional we first perform the following change of variables (cutoff BRS) in (15)

$$
\begin{aligned}
\delta A_{\mu} & =\eta \partial_{\mu} \bar{c}, \quad \delta \bar{c}=0, \quad \delta c=\eta \frac{1}{\alpha}\left(\partial A-\alpha M \phi_{2}\right), \\
\delta \phi_{1} & =-\eta K_{0 \Lambda} \frac{\delta S_{\mathrm{eff}}}{\delta \chi_{1}}, \quad \delta \phi_{2}=\eta\left(M \bar{c}-K_{0 \Lambda} \frac{\delta S_{\mathrm{eff}}}{\delta \chi_{2}}\right) .
\end{aligned}
$$

One then deduces the following identity

$$
\mathcal{S} e^{-W}=N \int \mathcal{D} \phi \Delta_{\text {eff }} \exp \left\{\left(-S_{2}+S_{J}+\chi_{2} M \bar{c}\right)_{0 \Lambda}-S_{\text {eff }}\right\}
$$

which gives the violation of the ST identities. The violation $\Delta_{\text {eff }}=\Delta_{1}+\Delta_{2}$ is

$$
\begin{gathered}
\Delta_{1}=\left[-\phi_{1} D_{1}^{-1} \frac{\delta}{\delta \chi_{1}}-\phi_{2} D_{2}^{-1} \frac{\delta}{\delta \chi_{2}}+\left(\partial_{\mu} \bar{c}\right) \frac{\delta}{\delta A_{\mu}}+M \bar{c} \frac{\delta}{\delta \phi_{2}}+\frac{1}{\alpha}\left(\partial_{\mu} A_{\mu}-\alpha M \phi_{2}\right) \frac{\delta}{\delta c}\right] S_{\mathrm{eff}} \\
\Delta_{2}=\int_{p} K_{0 \Lambda}(p) \sum_{i=1,2}\left(\frac{\delta^{2} S_{\mathrm{eff}}}{\delta \phi_{i} \delta \chi_{i}}-\frac{\delta S_{\mathrm{eff}}}{\delta \phi_{i}} \frac{\delta S_{\mathrm{eff}}}{\delta \chi_{i}}\right) .
\end{gathered}
$$

We now discuss the conditions needed to have $\Delta_{\text {eff }}=0$, i.e. the ST identities.

The operator $\Delta_{\text {eff }}$ satisfies a linear evolution equation which perturbatively has the form

$$
\Lambda \partial_{\Lambda} \Delta^{(n)}=L\left[\Delta^{(m)}\right], \quad m<n,
$$

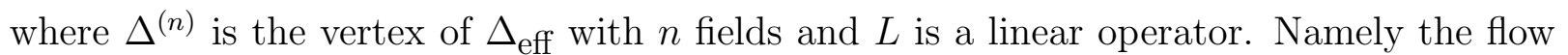
of a vertex of $\Delta_{\text {eff }}$ is given by the vertices with lower number of fields. From the linearity of this equation if one has vanishing boundary conditions then $\Delta_{\text {eff }}$ is zero for any $\Lambda$.

In order to discuss the boundary conditions we distinguish the relevant part, corresponding to the local approximant with monomials of dimension not larger than five and with ghost number one (see appendix C), and the remaining irrelevant part. From (16)

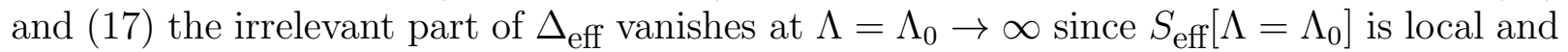
$K_{0 \infty}=1$. We now consider the boundary conditions for the relevant part. Since we are interested in fixing the relevant couplings of the effective action at the physical point, the vanishing of the relevant parameters of $\Delta_{\text {eff }}$ is imposed at $\Lambda=0$. Moreover at this point 
the functional $\Delta_{\text {eff }}$ is simpler since $\Delta_{2}=0$ and $\Delta_{1}$ becomes by Legendre transform the ST functional $\Delta_{\Gamma} \equiv \mathcal{S}_{\Gamma} \Gamma$. As a consequence of the above considerations we have [7]

$$
\Delta_{\Gamma, \mathrm{rel}}=0 \quad \Rightarrow \quad \Delta_{\Gamma}=0
$$

where $\Delta_{\Gamma, \text { rel }}$ is the relevant part of $\Delta_{\Gamma}$. It is a general feature that the requirement $\Delta_{\Gamma, \text { rel }}=0$ gives a number of conditions, the fine tuning conditions, larger than the number of couplings which have to be fixed. However due to the nilpotency of the BRS transformation one can find a set of algebraic consistency conditions which must be identically satisfied by $\Delta_{\Gamma}$. In our case one can see that

$$
\left[\mathcal{S}_{\Gamma}-\frac{1}{2} \sum_{i=1,2}\left(\frac{\delta \Gamma}{\delta \phi_{i}} \frac{\delta}{\delta \chi_{i}}+\frac{\delta \Gamma}{\delta \chi_{i}} \frac{\delta}{\delta \phi_{i}}\right)\right] \Delta_{\Gamma}=0 .
$$

This is a functional identity which mixes relevant and irrelevant parts of $\Delta_{\Gamma}$, due to the presence of mass scales (which can be the masses of the particles, as in our case, or the subtraction point in a massless theory). If one shows that these irrelevant parts vanish, the consistency conditions become a set of relations among the relevant couplings of $\Delta_{\Gamma}$ [16], thus giving a reduction of the number of independent relations in $\Delta_{\Gamma, \text { rel }}=0$.

In general at this point one invokes the so-called Quantum Action Principle (QAP) which states the locality of $\Delta_{\Gamma}$ at the first non-trivial loop order. However one can avoid the use of the QAP and exploit only the properties of the RG flow. As shown in [7] the locality of $\Delta_{\Gamma}$ can be recovered by solving the fine tuning conditions starting from the vertices of $\Delta_{\Gamma}$ with the lowest number of fields, i.e. $\Delta^{(2)}$.

We briefly summarize the proof. Due to the form of the evolution equation (18), perturbatively the vertex $\Delta^{(2)}$ does not flow. Therefore this vertex is given by its relevant part at $\Lambda=0$ and can be set to zero by tuning the couplings in the effective action. One then considers the evolution of the vertices $\Delta^{(3)}$. From eq. (18) and the fact that $\Delta^{(2)}=0$ these vertices do not flow. Therefore they are equal to their relevant parts at $\Lambda=0$. By using the consistency conditions one reduces the number of independent parameters of $\Delta^{(3)}$ and show that also $\Delta^{(3)}$ can be set to zero by fixing the couplings of $\Gamma$. This procedure can be repeated until the whole $\Delta_{\Gamma, \text { rel }}$ is fixed to zero. The technique we described is equivalent to the introduction of a filtration in the analysis of the cohomology of the ST operator [17].

The explicit solution of the various fine tuning equations is given in appendix D. After having fixed the following physical conditions

$$
\begin{gathered}
\sigma_{m_{A}}=M^{2}, \quad \sigma_{A}=1-\frac{1}{\alpha}, \quad \sigma_{\phi_{2} A}=0, \\
\sigma_{m_{1}}=m^{2}, \quad \sigma_{1}=1, \quad \sigma_{A \phi_{1} \phi_{2}}=-i e
\end{gathered}
$$

for the remaining couplings we use the ST identities. One finds

$$
\sigma_{\bar{c} \chi_{2}}=M, \quad \sigma_{m_{2}}=\alpha M^{2}, \quad \sigma_{2}=1 .
$$

All the other couplings receive perturbative corrections. As an example we now evaluate the one loop correction to the boundary condition on the gauge parameter $\sigma_{\alpha}$. This is fixed to be

$$
\sigma_{\alpha}=\frac{1}{\alpha}+\left.M \frac{\partial \Sigma^{\left(\bar{c} \chi_{2}\right)}(p)}{\partial p^{2}}\right|_{p^{2}=0}+\left.i M \frac{\partial \Sigma^{\left(\phi_{2} A\right)}(p)}{\partial p^{2}}\right|_{p^{2}=0}
$$


At one loop one finds in the Feynman gauge

$$
\begin{gathered}
\left.\frac{\partial \Sigma^{\left(\bar{c} \chi_{2}\right)}(p)}{\partial p^{2}}\right|_{p^{2}=0}=-e^{2} M m^{2} I_{3} \\
\left.\frac{\partial \Sigma^{\left(\phi_{2} A\right)}(p)}{\partial p^{2}}\right|_{p^{2}=0}=2 i e^{2} M m^{2}\left(I_{3}-m^{2} I_{4}\right)+i e^{2} \frac{m^{4}}{M}\left(I_{3}-2 m^{2} I_{4}\right),
\end{gathered}
$$

where $I_{3}$ and $I_{4}$ are given by

$$
\begin{gathered}
16 \pi^{2} I_{3}=\frac{M^{2}}{\left(m^{2}-M^{2}\right)^{3}} \log \frac{M^{2}}{m^{2}}+\frac{M^{2}+m^{2}}{2 m^{2}\left(m^{2}-M^{2}\right)^{2}}, \\
16 \pi^{2} I_{4}=\frac{M^{2}}{\left(m^{2}-M^{2}\right)^{4}} \log \frac{M^{2}}{m^{2}}+\frac{5 m^{2} M^{2}-M^{4}+2 m^{4}}{6 m^{4}\left(m^{2}-M^{2}\right)^{3}} .
\end{gathered}
$$

Then the one loop correction to the gauge parameter is

$$
\sigma_{\alpha}^{(1)}=e^{2} m^{2}\left[-\left(m^{2}+3 M^{2}\right) I_{3}+2 m^{2}\left(m^{2}+M^{2}\right) I_{4}\right]
$$

\section{Conclusions}

In this paper we have seen what are the boundary conditions that one has to impose on the RG flow in order to describe a theory with a spontaneously broken symmetry. We have discussed the global and the local case.

In the global case, the implementation of the symmetry, which is immediate in the unbroken phase, requires a non-trivial perturbative fine tuning of the boundary conditions. We considered the discrete $Z_{2}$ symmetry, but the analysis for a continuous one is identical.

In the local case the implementation of the symmetry can be performed along the same lines in both phases. The problem can be reduced to the vanishing of the relevant part of the Slavnov-Taylor identities. In principle one could expect that the broken phase is simpler, since the vector mass avoids problems related to infrared singularities and there is no need of introducing a non-vanishing subtraction point. For this reason for instance the extraction of the relevant part of a functional can be performed at vanishing momentum and reduces to a truncated Taylor expansion. In the massless case the non-vanishing subtraction point causes some technical problems in the solution of the fine tuning equation and the consistency conditions, since it mixes the relevant and irrelevant contributions of the various vertices. As a consequence the filtration of the operator $\Delta_{\Gamma}$ must be introduced. However we have found that also in the broken phase the solution of the fine tuning equations is quite complicated. In this case the mass terms mix the relevant and irrelevant contributions and a filtration of $\Delta_{\Gamma}$ is also needed.

For simplicity we have considered only the abelian Higgs model. The non-abelian case can be treated along the same lines. Obviously one should introduce a non-vanishing

subtraction point in order to define the relevant part of the vertices involving vector fields of the unbroken symmetry generators. 
In the $Z_{2}$ scalar case we have computed the one loop effective potential and found that in the direction in which the symmetry is broken the cutoff effective action acquires a $\Lambda$-dependent minimum which vanishes only for $\Lambda=0$. We computed the one-loop value of this running minimum for the scalar case. The analysis done for the scalar case (see eqs. (9)-(11) ) could be repeated for the Abelian Higgs model. In this case $v^{(1)}$ is the one loop correction to the parameter $v^{(0)}=M / e$. The qualitative results are the same, the only changes are in the factors. That is, at any non-vanishing $\Lambda$ the value $\phi_{1}=0$ is not the minimum of the cutoff effective potential. The running minimum is given by the one-point function of $\phi_{1}$.

Acknowledgements. We would like to thank C. Becchi, G. Bottazzi, for discussions and G. Marchesini and M. Pietroni for discussions and careful reading of part of the manuscript. M. D'A. would like to thank the LPTHE (Paris VI-VII) for kind hospitality.

\section{Appendix A}

In this appendix we perform some one loop computations for the scalar case. The evolution equation for the cutoff effective potential can be derived from (3). However we find more convenient to use the flow equation [18

$$
\Lambda \frac{\partial}{\partial \Lambda} V(\phi ; \Lambda)=-\frac{\not}{16 \pi^{2}} \Lambda^{4} \log \left(\Lambda^{2}+V^{\prime \prime}(\phi ; \Lambda)\right) .
$$

This equation can be obtained from (3) in the approximation in which the momentum dependent part of the vertices of the cutoff effective action is discarded (see 19 for the derivation). By solving this equation iteratively one obtains the loop expansion of $V$ in this approximation. Notice that in the one loop case (19) is exact since in the r.h.s. one must use the tree level value of $V^{\prime \prime}$ which is momentum independent?. In this case it is easy to integrate (19) with the boundary conditions (田) on the relevant couplings (and vanishing boundary conditions at $\Lambda=\Lambda_{0}$ for the irrelevant part). Thus the $\Lambda_{0} \rightarrow \infty$ limit can be taken and we get

$$
\begin{aligned}
& 64 \pi^{2} V^{(1)}[\phi ; \Lambda]=-\sqrt{3 g} m^{3} \phi-5 g m^{2} \phi^{2}+\left(\frac{64 \pi^{2}}{6} g_{3}^{(1)}-\frac{5 m g \sqrt{3 g}}{2}\right) \phi^{3}-\frac{9 g^{2}}{8} \phi^{4} \\
& -\frac{g}{2} \Lambda^{2}\left(\phi+v^{(0)}\right)^{2}+\left[\left(\frac{g}{2}\left(\phi+v^{(0)}\right)^{2}-\frac{m^{2}}{2}\right)^{2}-\Lambda^{4}\right] \log \left(\frac{\Lambda^{2}+\frac{g}{2}\left(\phi+v^{(0)}\right)^{2}-\frac{m^{2}}{2}}{m^{2}}\right),
\end{aligned}
$$

apart from a field independent term. In this expression the constant $g_{3}^{(1)}$ is the boundary condition for the three point coupling and has to be determined by using (7) in terms of the irrelevant part $V_{\text {irr }}^{(1)}(\phi ; \Lambda=0)$ of this one loop potential at $\Lambda=0$. In order to isolate this functional one notices that at $\Lambda=0$ the relevant part of (20) is simply given by

$$
V_{\mathrm{rel}}^{(1)}[\phi ; \Lambda=0]=\frac{1}{6} g_{3}^{(1)} \phi^{3},
$$

\footnotetext{
${ }^{2}$ For the Abelian Higgs model a formula analogous to (19) gives the exact one loop effective potential only in the Landau gauge, since in this gauge the derivative couplings do not contribute to the scalar sector.
} 
thus one obtains $V_{\text {irr }}^{(1)}(\phi ; \Lambda=0)$ by setting $g_{3}^{(1)}=0$ and $\Lambda=0$ in (20). By using this result in (4)-(8) one gets (9). The effective potential (20) is now completely determined and given by (10). For instance the one loop relevant couplings are

$$
\begin{gathered}
\rho^{(1)}(\Lambda)=\frac{\sqrt{3 g} m}{32 \pi^{2}}\left[-\Lambda^{2}+m^{2} \log \left(\frac{\Lambda^{2}+m^{2}}{m^{2}}\right)\right], \\
\sigma^{(1)}(\Lambda)=\frac{g}{32 \pi^{2}}\left[-\Lambda^{2}-\frac{3 m^{2} \Lambda^{2}}{\Lambda^{2}+m^{2}}+4 m^{2} \log \left(\frac{\Lambda^{2}+m^{2}}{m^{2}}\right)\right], \\
g_{3}^{(1)}(\Lambda)=\frac{3 m g \sqrt{3 g}}{32 \pi^{2}}\left[-\frac{5 \Lambda^{4}+4 m^{2} \Lambda^{2}+m^{4}}{2\left(\Lambda^{2}+m^{2}\right)^{2}}+\log \left(\frac{\Lambda^{2}+m^{2}}{m^{2}}\right)\right], \\
g^{(1)}(\Lambda)=\frac{3 g^{2}}{32 \pi^{2}}\left[-\frac{4 \Lambda^{6}-m^{2} \Lambda^{4}+m^{4} \Lambda^{2}}{\left(\Lambda^{2}+m^{2}\right)^{3}}+\log \left(\frac{\Lambda^{2}+m^{2}}{m^{2}}\right)\right] .
\end{gathered}
$$

In the limit $\Lambda \rightarrow \infty$ one obtains the one loop values of the "bare" couplings

$$
\begin{gathered}
\rho_{B}^{(1)}=\frac{\sqrt{3 g} m}{32 \pi^{2}}\left(-\Lambda^{2}+m^{2} T\right), \quad \sigma_{B}^{(1)}=\frac{g}{32 \pi^{2}}\left(-\Lambda^{2}-3 m^{2}+4 m^{2} T\right), \\
g_{3 B}^{(1)}=\frac{3 m g \sqrt{3 g}}{32 \pi^{2}}\left(-\frac{5}{2}+T\right), \quad g_{B}^{(1)}=\frac{3 g^{2}}{32 \pi^{2}}(-4+T),
\end{gathered}
$$

where $T=\log \left(\Lambda^{2} / m^{2}\right)$. Then the bare potential in terms of the unphysical field $\phi_{0}$ is

$$
\frac{1}{2}\left[-\frac{m^{2}}{2}+\frac{\not h g}{64 \pi^{2}}\left(-2 \Lambda^{2}+3 m^{2}-m^{2} T\right)\right] \phi_{0}^{2}+\frac{1}{4 !}\left[g+\frac{3 \not h g^{2}}{32 \pi^{2}}(-4+T)\right] \phi_{0}^{4} .
$$

\section{Appendix B}

Here we explicitly give the relevant part of the effective action $\Gamma[\Phi, \chi]$. We use the fact that under charge conjugation the functional $\Gamma[\Phi, \chi]$ is even. Recall that under charge conjugation $\phi_{1}, \bar{c}$ and $\chi_{2}$ are even while $A_{\mu}, \phi_{2}$ and $\chi_{1}$ are odd.

Using the equation of motion for the ghost $c$, the contributions to $\Gamma[\Phi, \chi]$ which contain 
relevant couplings are

$$
\begin{aligned}
& \Gamma[\Phi, \chi]=\frac{1}{2} \int_{p}\left\{\Gamma_{\mu \nu}^{(A A)}(p) A_{\mu}(-p) A_{\nu}(p)+\Gamma^{\left(\phi_{1} \phi_{1}\right)}(p) \phi_{1}(-p) \phi_{1}(p)+\Gamma^{\left(\phi_{2} \phi_{2}\right)}(p) \phi_{2}(-p) \phi_{2}(p)\right. \\
& \left.+2 \Gamma_{\mu}^{\left(\phi_{2} A\right)}(p) \phi_{2}(-p) A_{\mu}(p)+2 \Gamma^{\left(\bar{c} \chi_{2}\right)}(p) \bar{c}(-p) \chi_{2}(p)\right\} \\
& +\int_{p} \int_{q}\left\{\frac{1}{2} \Gamma_{\mu \nu}^{\left(2 A \phi_{1}\right)}(p, q, r) A_{\mu}(p) A_{\nu}(q) \phi_{1}(r)+\Gamma_{\mu}^{\left(A \phi_{1} \phi_{2}\right)}(p, q, r) A_{\mu}(p) \phi_{1}(q) \phi_{2}(r)\right. \\
& +\frac{1}{3 !} \Gamma^{\left(3 \phi_{1}\right)}(p, q, r) \phi_{1}(p) \phi_{1}(q) \phi_{1}(r)+\frac{1}{2} \Gamma^{\left(\phi_{1} 2 \phi_{2}\right)}(p, q, r) \phi_{1}(p) \phi_{2}(q) \phi_{2}(r) \\
& \left.+\Gamma^{\left(\bar{c} \chi_{1} \phi_{2}\right)}(p, q, r) \bar{c}(p) \chi_{1}(q) \phi_{2}(r)+\Gamma^{\left(\bar{c} \chi_{2} \phi_{1}\right)}(p, q, r) \bar{c}(p) \chi_{2}(q) \phi_{1}(r)\right\} \\
& +\frac{1}{4 !} \int_{p} \int_{q} \int_{k}\left\{\Gamma^{\left(4 \phi_{1}\right)}(p, q, k, h) \phi_{1}(p) \phi_{1}(q) \phi_{1}(k) \phi_{1}(h)+\Gamma^{\left(4 \phi_{2}\right)}(p, q, k, h) \phi_{2}(p) \phi_{2}(q) \phi_{2}(k) \phi_{2}(h)\right. \\
& +6 \Gamma^{\left(2 \phi_{1} 2 \phi_{2}\right)}(p, q, k, h) \phi_{1}(p) \phi_{1}(q) \phi_{2}(k) \phi_{2}(h)+6 \Gamma_{\mu \nu}^{\left(2 \phi_{1} 2 A\right)}(p, q, k, h) \phi_{1}(p) \phi_{1}(q) A_{\mu}(k) A_{\nu}(h) \\
& \left.+6 \Gamma_{\mu \nu}^{\left(2 \phi_{2} 2 A\right)}(p, q, k, h) \phi_{2}(p) \phi_{2}(q) A_{\mu}(k) A_{\nu}(h)+\Gamma_{\mu \nu \rho \sigma}^{(4 A)}(p, q, k, h) A_{\mu}(p) A_{\nu}(q) A_{\rho}(k) A_{\sigma}(h)\right\} \\
& +\ldots,
\end{aligned}
$$

where $r=-p-q, h=-p-q-k$ and the dots stand for all the remaining terms which contain only irrelevant vertices, since they are coefficients of monomials in the fields and sources with dimension higher than four.

The vertices in (21) contain 22 relevant couplings which are defined as follows

$$
\begin{aligned}
& \Gamma_{\mu \nu}^{(A A)}(p)=\delta_{\mu \nu}\left[\sigma_{m_{A}}+p^{2} \sigma_{\alpha}+\Sigma_{L}(p)\right]+t_{\mu \nu}(p)\left[\sigma_{A}+\Sigma_{T}(p)\right] \\
& \Gamma^{\left(\phi_{1} \phi_{1}\right)}(p)=\sigma_{m_{1}}+p^{2} \sigma_{1}+\Sigma_{1}(p) \\
& \Gamma^{\left(\phi_{2} \phi_{2}\right)}(p)=\sigma_{m_{2}}+p^{2} \sigma_{2}+\Sigma_{2}(p) \\
& \Gamma_{\mu}^{\left(\phi_{2} A\right)}(p)=p_{\mu}\left(\sigma_{\phi_{2} A}+\Sigma^{\left(\phi_{2} A\right)}(p)\right) \\
& \Gamma^{\left(\bar{c} \chi_{2}\right)}(p)=\sigma_{\bar{c} \chi_{2}}+\Sigma^{\left(\bar{c} \chi_{2}\right)}(p) \\
& \Gamma_{\mu \nu}^{\left(2 A \phi_{1}\right)}(p, q, r)=\delta_{\mu \nu}\left[\sigma_{2 A \phi_{1}}+\Sigma^{\left(2 A \phi_{1}\right)}(p, q, r)\right]+\tilde{\Gamma}_{\mu \nu}^{\left(2 A \phi_{1}\right)}(p, q, r) \\
& \Gamma_{\mu}^{\left(A \phi_{1} \phi_{2}\right)}(p, q, r)=q_{\mu}\left[\sigma_{A \phi_{1} \phi_{2}}+\Sigma^{\left(A \phi_{1} \phi_{2}\right)}(p, q, r)\right]+r_{\mu}\left[\sigma_{A \phi_{1} \phi_{2}}^{\prime}+\Sigma^{\left(A \phi_{1} \phi_{2}\right)}(p, q, r)\right], \\
& \Gamma^{\left(3 \phi_{1}\right)}(p, q, r)=\sigma_{3 \phi_{1}}+\Sigma^{\left(3 \phi_{1}\right)}(p, q, r), \\
& \Gamma^{\left(\phi_{1} 2 \phi_{2}\right)}(p, q, r)=\sigma_{\phi_{1} 2 \phi_{2}}+\Sigma^{\left(\phi_{1} 2 \phi_{2}\right)}(p, q, r), \\
& \Gamma^{\left(\bar{c} \chi_{1} \phi_{2}\right)}(p, q, r)=\sigma_{\bar{c} \chi_{1} \phi_{2}}+\Sigma^{\left(\bar{c} \chi_{1} \phi_{2}\right)}(p, q, r), \\
& \Gamma^{\left(\bar{c} \chi_{2} \phi_{1}\right)}(p, q, r)=\sigma_{\bar{c} \chi_{2} \phi_{1}}+\Sigma^{\left(\bar{c} \chi_{2} \phi_{1}\right)}(p, q, r), \\
& \Gamma^{\left(4 \phi_{1}\right)}(p, q, k, h)=\sigma_{4 \phi_{1}}+\Sigma^{\left(4 \phi_{1}\right)}(p, q, k, h), \\
& \Gamma^{\left(4 \phi_{2}\right)}(p, q, k, h)=\sigma_{4 \phi_{2}}+\Sigma^{\left(4 \phi_{2}\right)}(p, q, k, h), \\
& \Gamma^{\left(2 \phi_{1} 2 \phi_{2}\right)}(p, q, k, h)=\sigma_{2 \phi_{1} 2 \phi_{2}}+\Sigma^{\left(2 \phi_{1} 2 \phi_{2}\right)}(p, q, k, h), \\
& \Gamma_{\mu \nu}^{\left(2 \phi_{1} 2 A\right)}(p, q, k, h)=\delta_{\mu \nu}\left[\sigma_{2 \phi_{1} 2 A}+\Sigma^{\left(2 \phi_{1} 2 A\right)}(p, q, k, h)\right]+\tilde{\Gamma}_{\mu \nu}^{\left(2 \phi_{1} 2 A\right)}(p, q, k, h), \\
& \Gamma_{\mu \nu}^{\left(2 \phi_{2} 2 A\right)}(p, q, k, h)=\delta_{\mu \nu}\left[\sigma_{2 \phi_{2} 2 A}+\Sigma^{\left(2 \phi_{2} 2 A\right)}(p, q, k, h)\right]+\tilde{\Gamma}_{\mu \nu}^{\left(2 \phi_{2} 2 A\right)}(p, q, k, h), \\
& \Gamma_{\mu \nu \rho \sigma}^{(4 A)}(p, q, k, h)=\left(\delta_{\mu \nu} \delta_{\rho \sigma}+\delta_{\mu \rho} \delta_{\nu \sigma}+\delta_{\mu \sigma} \delta_{\nu \rho}\right)\left[\sigma_{4 A}+\Sigma^{(4 A)}(p, q, k, h)\right]+\tilde{\Gamma}_{\mu \nu \rho \sigma}^{(4 A)}(p, q, k, h),
\end{aligned}
$$


with the conditions

$$
\begin{gathered}
\Sigma_{L}(0)=0,\left.\quad \frac{\partial \Sigma_{L}(p)}{\partial p^{2}}\right|_{p^{2}=0}=0,\left.\quad \Sigma_{T}(p)\right|_{p^{2}=0}=0, \\
\Sigma_{i}(0)=0,\left.\quad \frac{\partial \Sigma_{i}(p)}{\partial p^{2}}\right|_{p^{2}=0}=0, \quad i=1,2, \\
\left.\Sigma^{(\cdots)}\left(p_{i}\right)\right|_{p_{i}=0}=0 .
\end{gathered}
$$

In the various $\Sigma$ we can factorize a dimensional function of $p$. Thus they are irrelevant and contribute to the irrelevant part of the functional $\Gamma[\Phi, \chi]$. Similarly the vertices $\tilde{\Gamma}_{i}$ are irrelevant since their Lorentz structure is (partially in the case of $\tilde{\Gamma}^{(4 A)}$ ) given by external momenta.

We recall that the ghost propagator and the $\bar{c}-c-\phi_{1}$ vertex are given in terms of the vertices $\Gamma^{\left(\bar{c} \chi_{2}\right)}$ and $\Gamma^{\left(\bar{c} \chi_{2} \phi_{1}\right)}$ by

$$
\Gamma^{(\bar{c} c)}(p)=-p^{2}-\alpha M \Gamma^{\left(\bar{c} \chi_{2}\right)}(p), \quad \Gamma^{\left(\bar{c} c \phi_{1}\right)}=-\alpha M \Gamma^{\left(\bar{c} \chi_{2} \phi_{1}\right)} .
$$

At tree level one has

$$
\Gamma^{(\bar{c} c)}(p)=-\left(p^{2}+\alpha M^{2}\right)
$$

and

$$
\Gamma^{\left(\bar{c} c \phi_{1}\right)}(p, q, r)=-\alpha M e .
$$

\section{Appendix $\mathrm{C}$}

We now extract the relevant part of the most general one dimensional functional of fields and sources with ghost number -1 and odd under charge conjugation. We call this generic functional $\Delta$. The vertices of $\Delta$ which contain the relevant couplings come from the $\Delta^{(n)}$ with $n=2, \ldots 5$, where $n$ denotes the number of fields. From the two-fields component

$$
\Delta^{(2)}=\int_{p}\left\{\Delta_{\mu}^{(\bar{c} A)}(p) \bar{c}(-p) A_{\mu}(p)+\Delta^{\left(\bar{c} \phi_{2}\right)}(p) \bar{c}(-p) \phi_{2}(p)\right\},
$$

we have the following relevant parameters

$$
\begin{aligned}
\Delta_{\mu}^{(\bar{c} A)}(p) & =p_{\mu}\left[\delta_{1}+p^{2} \delta_{2}+\Delta_{\text {irr }}^{(\bar{c} A)}(p)\right], \\
\Delta^{\left(\bar{c} \phi_{2}\right)}(p) & =\delta_{3}+p^{2} \delta_{4}+\Delta_{\text {irr }}^{\left(\bar{c} \phi_{2}\right)}(p) .
\end{aligned}
$$

The three-fields component is

$$
\Delta^{(3)}=\int_{p} \int_{q}\left\{\Delta_{\mu}^{\left(\bar{c} A \phi_{1}\right)}(p, q, r) \bar{c}(p) A_{\mu}(q) \phi_{1}(r)+\Delta^{\left(\bar{c} \phi_{1} \phi_{2}\right)}(p, q, r) \bar{c}(p) \phi_{1}(q) \phi_{2}(r)\right\}+\ldots,
$$

where $r=-p-q$ and the dots stand for the remaining terms which are all irrelevant. It contains the following relevant parameters

$$
\begin{aligned}
\Delta_{\mu}^{\left(\bar{c} A \phi_{1}\right)}(p, q, r) & =p_{\mu}\left[\delta_{5}+\Delta_{1, \mathrm{irr}}^{\left(\bar{c} A \phi_{1}\right)}(p, q, r)\right]+q_{\mu}\left[\delta_{6}+\Delta_{2, \mathrm{irr}}^{\left(\bar{c} A \phi_{1}\right)}(p, q, r)\right], \\
\Delta^{\left(\bar{c} \phi_{1} \phi_{2}\right)}(p, q, r) & =\delta_{7}+q^{2} \delta_{8}+r^{2} \delta_{9}+q \cdot r \delta_{10}+\Delta_{\mathrm{irr}}^{\left(\bar{c} \phi_{1} \phi_{2}\right)}(p, q, r) .
\end{aligned}
$$


From the four-fields component

$$
\begin{aligned}
\Delta^{(4)} & =\int_{p} \int_{q} \int_{k}\left\{\Delta_{\mu \nu \rho}^{(\bar{c} 3 A)}(p, q, k, h) \bar{c}(p) A_{\mu}(q) A_{\nu}(k) A_{\rho}(h)\right. \\
& +\Delta_{\mu \nu}^{\left(\bar{c} 2 A \phi_{2}\right)}(p, q, k, h) \bar{c}(p) A_{\mu}(q) A_{\nu}(k) \phi_{2}(h)+\Delta_{\mu}^{\left(\bar{c} A 2 \phi_{1}\right)}(p, q, k, h) \bar{c}(p) A_{\mu}(q) \phi_{1}(k) \phi_{1}(h) \\
& +\Delta_{\mu}^{\left(\bar{c} A 2 \phi_{2}\right)}(p, q, k, h) \bar{c}(p) A_{\mu}(q) \phi_{2}(k) \phi_{2}(h)+\Delta^{\left(\bar{c} 3 \phi_{2}\right)}(p, q, k, h) \bar{c}(p) \phi_{2}(q) \phi_{2}(k) \phi_{2}(h) \\
& \left.+\Delta^{\left(\bar{c} 2 \phi_{1} \phi_{2}\right)}(p, q, k, h) \bar{c}(p) \phi_{1}(q) \phi_{1}(k) \phi_{2}(h)\right\}+\cdots,
\end{aligned}
$$

where $h=-p-q-k$, we have the relevant parameters

$$
\begin{aligned}
\Delta_{\mu \nu \rho}^{(\bar{c} 3 A)}(p, q, k, h) & =\left(q_{\mu} \delta_{\nu \rho}+k_{\nu} \delta_{\mu \rho}+h_{\rho} \delta_{\mu \nu}\right)\left[\delta_{11}+\Delta_{1, \mathrm{irr}}^{(\bar{c} 3 A)}(p, q, k, h)\right] \\
& +\left[(k+h)_{\mu} \delta_{\nu \rho}+(q+k)_{\rho} \delta_{\mu \nu}+(q+h)_{\nu} \delta_{\mu \rho}\right]\left[\delta_{12}+\Delta_{2, \mathrm{irr}}^{(\bar{c} 3 A)}(p, q, k, h)\right] \\
& +\tilde{\Delta}_{\mu \nu \rho}^{(\bar{c} 3 A)}(p, q, k, h) \\
\Delta_{\mu \nu}^{\left(\bar{c} 2 A \phi_{2}\right)}(p, q, k, h) & =\delta_{\mu \nu}\left[\delta_{13}+\Delta_{\text {irr }}^{\left(\bar{c} 2 A \phi_{2}\right)}(p, q, k, h)\right]+\tilde{\Delta}_{\mu \nu}^{\left(\bar{c} 2 A \phi_{2}\right)}(p, q, k, h), \\
\Delta_{\mu}^{\left(\bar{c} A 2 \phi_{1}\right)}(p, q, k, h) & =p_{\mu}\left[\delta_{14}+\Delta_{1, \mathrm{irr}}^{\left(\bar{c} A 2 \phi_{1}\right)}(p, q, k, h)\right]+q_{\mu}\left[\delta_{15}+\Delta_{2, \mathrm{irr}}^{\left(\bar{c} A 2 \phi_{1}\right)}(p, q, k, h)\right], \\
\Delta_{\mu}^{\left(\bar{c} A 2 \phi_{2}\right)}(p, q, k, h) & =p_{\mu}\left[\delta_{16}+\Delta_{1, \mathrm{irr}}^{\left(\bar{c} A 2 \phi_{2}\right)}(p, q, k, h)\right]+q_{\mu}\left[\delta_{17}+\Delta_{2, \mathrm{irr}}^{\left(\bar{c} A 2 \phi_{2}\right)}(p, q, k, h)\right], \\
\Delta^{\left(\bar{c} 3 \phi_{2}\right)}(p, q, k, h) & =\delta_{18}+\Delta_{\mathrm{irr}}^{\left(\bar{c} 3 \phi_{2}\right)}(p, q, k, h), \\
\Delta^{\left(\bar{c} 2 \phi_{1} \phi_{2}\right)}(p, q, k, h) & =\delta_{19}+\Delta_{\mathrm{irr}}^{\left(\bar{c} 2 \phi_{1} \phi_{2}\right)}(p, q, k, h) .
\end{aligned}
$$

Finally the five-fields component

$$
\begin{aligned}
\Delta^{(5)} & =\int_{p} \int_{q} \int_{k} \int_{h}\left\{\Delta_{\mu \nu}^{\left(\bar{c} 2 A \phi_{1} \phi_{2}\right)}(p, q, k, h, s) \bar{c}(p) A_{\mu}(q) A_{\nu}(k) \phi_{1}(h) \phi_{2}(s)\right. \\
& +\Delta^{\left(\bar{c} 3 \phi_{1} \phi_{2}\right)}(p, q, k, h, s) \bar{c}(p) \phi_{1}(q) \phi_{1}(k) \phi_{1}(h) \phi_{2}(s) \\
& \left.+\Delta^{\left(\bar{c} \phi_{1} 3 \phi_{2}\right)}(p, q, k, h, s) \bar{c}(p) \phi_{1}(q) \phi_{2}(k) \phi_{2}(h) \phi_{2}(s)\right\}+\ldots,
\end{aligned}
$$

where $s=-p-q-k-h$, contains the parameters

$$
\begin{aligned}
\Delta_{\mu \nu}^{\left(\bar{c} 2 A \phi_{1} \phi_{2}\right)}(p, q, k, h, s) & =\delta_{\mu \nu}\left[\delta_{20}+\Delta_{\text {irr }}^{\left(\bar{c} 2 A \phi_{1} \phi_{2}\right)}(p, q, k, h, s)\right]+\tilde{\Delta}_{\mu \nu}^{\left(\bar{c} 2 A \phi_{1} \phi_{2}\right)}(p, q, k, h, s), \\
\Delta^{\left(\bar{c} 3 \phi_{1} \phi_{2}\right)}(p, q, k, h, s) & =\delta_{21}+\Delta_{\text {irr }}^{\left(\bar{c} 3 \phi_{1} \phi_{2}\right)}(p, q, k, h, s), \\
\Delta^{\left(\bar{c} \phi_{1} 3 \phi_{2}\right)}(p, q, k, h, s) & =\delta_{22}+\Delta_{\text {irr }}^{\left(\bar{c} \phi_{1} 3 \phi_{2}\right)}(p, q, k, h, s) .
\end{aligned}
$$

The conditions defining the 22 relevant parameters are

$$
\Delta_{\text {irr }}^{(\bar{c} A)}(0)=\left.\frac{\partial}{\partial p^{2}} \Delta_{\text {irr }}^{(\bar{c} A)}(p)\right|_{p^{2}=0}=0, \quad \Delta_{\text {irr }}^{\left(\bar{c} \phi_{2}\right)}(0)=\left.\frac{\partial}{\partial p^{2}} \Delta_{\text {irr }}^{\left(\bar{c} \phi_{2}\right)}(p)\right|_{p^{2}=0}=0
$$

and

$$
\left.\Delta_{\text {irr }}^{(\bar{c} \cdots)}(p, \ldots)\right|_{p_{i}=0}=0
$$

for the other vertices.

Due to these conditions one can isolate in these vertices a dimensional function of the momenta thus they are irrelevant. Similarly the vertices $\tilde{\Delta}_{i}$ have the Lorentz indices carried by momenta in a different way with respect to their relevant parts and are irrelevant. 


\section{Appendix D}

In this appendix we perform the fine tuning of the couplings of the effective action. The condition $\Delta^{(2)}=0$ gives

$$
\begin{aligned}
& \delta_{1}=0 \rightarrow \sigma_{\bar{c} \chi_{2}}=M, \\
& \delta_{2}=0 \rightarrow \sigma_{\alpha}=\frac{1}{\alpha}+M\left(\left.\frac{\partial \Sigma^{\left(\bar{c} \chi_{2}\right)}}{\partial p^{2}}\right|_{p^{2}=0}+\left.i \frac{\partial \Sigma^{\left(\phi_{2} A\right)}}{\partial p^{2}}\right|_{p^{2}=0}\right), \\
& \delta_{3}=0 \rightarrow \sigma_{m_{2}}=\alpha M^{2}, \\
& \delta_{4}=0 \rightarrow \sigma_{2}=1 .
\end{aligned}
$$

The condition $\Delta^{(3)}=0$ gives

$$
\begin{aligned}
& \delta_{5}=0 \rightarrow i \sigma_{2 A \phi_{1}}-M \sigma_{A \phi_{1} \phi_{2}}^{\prime}=i e M+m^{2} A(0), \\
& \delta_{6}=0 \rightarrow M \sigma_{\bar{c} \chi_{2} \phi_{1}}=e M-i m^{2} B(0), \\
& \delta_{7}=0 \rightarrow M \sigma_{\phi_{1} 2 \phi_{2}}=-m^{2} \sigma_{\bar{c} \chi_{1} \phi_{2}}, \\
& \delta_{8}=0 \rightarrow \sigma_{\bar{c} \chi_{1} \phi_{2}}+\left.\sigma_{\phi_{1} 2 \phi_{2}} \frac{\partial \Sigma^{\left(\bar{c} \chi_{2}\right)}}{\partial p^{2}}\right|_{p^{2}=0}=-e-M C(0)-m^{2} E(0), \\
& \delta_{9}=0 \rightarrow i \sigma_{A \phi_{1} \phi_{2}}^{\prime}=M D(0)-2 M C(0)-\sigma_{\bar{c} \chi_{2} \phi_{1}}-\left.\sigma_{\phi_{1} 2 \phi_{2}} \frac{\partial \Sigma^{\left(\bar{c} \chi_{2}\right)}}{\partial p^{2}}\right|_{p^{2}=0}-m^{2} F(0)
\end{aligned}
$$

and $\delta_{10}$ is zero due to a consistency condition.

The condition $\Delta^{(4)}=0$ gives

$$
\begin{aligned}
& \delta_{12}=0 \rightarrow i \sigma_{4 A}=-M I(0)+[A(0)-B(0)] \sigma_{2 A \phi_{1}}, \\
& \delta_{13}=0 \rightarrow M \sigma_{2 A 2 \phi_{2}}+\sigma_{2 A \phi_{1}} \sigma_{\bar{c} \chi_{1} \phi_{2}}=0, \\
& \delta_{14}=0 \rightarrow i \sigma_{2 A 2 \phi_{1}}=\sigma_{3 \phi_{1}} A(0)+\left(\sigma_{A \phi_{1} \phi_{2}}^{\prime}+i e\right) \sigma_{\bar{c} \chi_{2} \phi_{1}}+M J(0), \\
& \delta_{15}=0 \rightarrow \sigma_{3 \phi_{1}} B(0)=\left(\sigma_{A \phi_{1} \phi_{2}}^{\prime}-i e\right) \sigma_{\bar{c} \chi_{2} \phi_{1}}-i M \Gamma^{\left(\bar{c} \chi_{2} 2 \phi_{1}\right)}\left(p_{i}=0\right)-M K(0), \\
& \delta_{18}=0 \rightarrow M \sigma_{4 \phi_{2}}+3 \sigma_{\phi_{1} 2 \phi_{2}} \sigma_{\bar{c} \chi_{2} \phi_{1}}=0, \\
& \delta_{19}=0 \rightarrow M \sigma_{2 \phi_{1} 2 \phi_{2}}+\sigma_{3 \phi_{1}} \sigma_{\bar{c} \chi_{1} \phi_{2}}+2 \sigma_{\phi_{1} 2 \phi_{2}} \sigma_{\bar{c} \chi_{2} \phi_{1}}=0
\end{aligned}
$$

and $\delta_{11}, \delta_{16}$ and $\delta_{17}$ are zero due to consistency conditions.

The condition $\Delta^{(5)}=0$ gives

$$
\begin{aligned}
& \delta_{21}=0 \rightarrow \sigma_{4 \phi_{1}} \sigma_{\bar{c} \chi_{1} \phi_{2}}=-3 \sigma_{2 \phi_{1} 2 \phi_{2}} \sigma_{\bar{c} \chi_{2} \phi_{1}}-3 m^{2} \Gamma^{\left(\bar{c} \chi_{1} 2 \phi_{1} \phi_{2}\right)}\left(p_{i}=0\right) \\
& -3 \sigma_{3 \phi_{1}} \Gamma^{\left(\bar{c} \chi_{1} \phi_{1} \phi_{2}\right)}\left(p_{i}=0\right)-3 \sigma_{\phi_{1} 2 \phi_{2}} \Gamma^{\left(\bar{c} \chi_{2} 2 \phi_{1}\right)}\left(p_{i}=0\right)-M \Gamma^{\left(3 \phi_{1} 2 \phi_{2}\right)}\left(p_{i}=0\right) \text {, }
\end{aligned}
$$

and $\delta_{20}$ and $\delta_{22}$ are zero due to consistency conditions.

The above solutions are written in terms of some form factors of the following irrelevant vertices

$$
\begin{gathered}
\Gamma_{\mu}^{\left(\bar{c} \chi_{1} A\right)}(p, q, k)=p_{\mu} A+k_{\mu} B, \quad \Sigma^{\left(\phi_{1} 2 \phi_{2}\right)}(p, q, k)=\left(q^{2}+k^{2}\right) C+(q k) D, \\
\Sigma^{\left(\bar{c} \chi_{1} \phi_{2}\right)}(p, q, k)=q^{2} E+k^{2} F+(q k) G, \quad \Gamma_{\mu}^{\left(2 \phi_{1} \phi_{2} A\right)}(p, q, k, h)=p_{\mu} J+q_{\mu} K,
\end{gathered}
$$




$$
\begin{aligned}
\Gamma_{\mu \nu \rho}^{\left(\phi_{2} 3 A\right)}(p, q, k, h)=\left(q_{\mu} \delta_{\nu \rho}+k_{\nu} \delta_{\mu \rho}\right. & \left.+h_{\rho} \delta_{\mu \nu}\right) H+\left[(k+h)_{\mu} \delta_{\nu \rho}+(q+k)_{\rho} \delta_{\mu \nu}+(q+h)_{\nu} \delta_{\mu \rho}\right] I \\
& +\tilde{\Gamma}_{\mu \nu \rho}^{\left(\phi_{2} 3 A\right)}(p, q, k, h) .
\end{aligned}
$$

At tree level one has

$$
\begin{gathered}
\sigma_{m_{A}}=M^{2}, \quad \sigma_{\alpha}=\frac{1}{\alpha}, \quad \sigma_{A}=1-\frac{1}{\alpha}, \\
\sigma_{m_{1}}=m^{2}, \quad \sigma_{1}=1, \quad \sigma_{m_{2}}=\alpha M^{2}, \quad \sigma_{2}=1, \\
\sigma_{\phi_{2} A}=0, \quad \sigma_{\bar{c} \chi_{2}}=M, \\
\sigma_{2 A \phi_{1}}=2 M e, \quad \sigma_{A \phi_{1} \phi_{2}}=-i e, \quad \sigma_{A \phi_{1} \phi_{2}}^{\prime}=i e \\
\sigma_{3 \phi_{1}}=3 ! M \frac{g}{e}, \quad \sigma_{\phi_{1} 2 \phi_{2}}=2 M \frac{g}{e}, \\
\sigma_{\bar{c} \chi_{1} \phi_{2}}=-e, \quad \sigma_{\bar{c} \chi_{2} \phi_{1}}=e, \\
\sigma_{4 \phi_{1}}=3 ! g, \quad \sigma_{4 \phi_{2}}=3 ! g, \quad \sigma_{2 \phi_{1} 2 \phi_{2}}=2 g \\
\sigma_{2 \phi_{1} 2 A}=2 e^{2}, \quad \sigma_{2 \phi_{2} 2 A}=2 e^{2}, \quad \sigma_{4 A}=0 .
\end{gathered}
$$

\section{References}

[1] T. Marinucci and M. Tonin, Il Nuovo Cimento 31A (1976) 381; S. Aoyama and M. Tonin, Nucl. Phys. B179 (1981) 293.

[2] R.J. Perry and K.G. Wilson, Nucl. Phys. B403 (1993) 587.

[3] K.G. Wilson, Phys. Rev. B 4 (1971) 3174, 3184; K.G. Wilson and J.G. Kogut, Phys. Rep. 12 (1974) 75.

[4] J. Polchinski, Nucl. Phys. B231 (1984) 269.

[5] G. Gallavotti, Rev. Mod. Phys. 57 (1985) 471.

[6] C. Becchi, On the construction of renormalized quantum field theory using renormalization group techniques, in Elementary particles, Field theory and Statistical mechanics, Eds. M. Bonini, G. Marchesini and E. Onofri, Parma University 1993.

[7] M. Bonini, M. D'Attanasio and G. Marchesini, Nucl. Phys. B437 (1995) 163, Phys. Lett. 346B (1995) 87.

[8] U. Ellwanger, Phys. Lett. 335B (1994) 364; U. Ellwanger, M. Hirsch and A. Weber, Flow equations for the relevant part of the pure Yang-Mills action, hep-th/9506019.

[9] B.J. Warr, Ann. Phys. (NY) 183 (1988) 1,89; G. Keller and C. Kopper, Phys. Lett. 273B (1991) 323; M. Reuter and C. Wetterich, Nucl. Phys. B417 (1994) 181.

[10] R. Floreanini and R. Percacci, The renormalization group flow of the dilaton potential, hep-th/9412181. 
[11] M. Bonini, M. D'Attanasio and G. Marchesini, Nucl. Phys. B409 (1993) 441, Nucl. Phys. B418 (1994) 81, Nucl. Phys. B421 (1994) 429.

[12] C. Wetterich, Phys. Lett. 301B (1993) 90; T.R. Morris, Int. J. Mod. Phys. A9 (1994) 2411.

[13] S. Coleman and E. Weinberg, Phys. Rev. D 7 (1973) 1888.

[14] D. Litim, N. Tetradis and C. Wetterich, Non-perturbative analysis of the ColemanWeinberg phase transition, hep-ph/9407267.

[15] C. Becchi, A. Rouet and R. Stora, Phys. Lett. 52B (1974) 344.

[16] C. Becchi, A. Rouet and R. Stora, Comm. Math. Phys. 42 (1975) 127.

[17] C. Becchi, A. Blasi, G. Bonneau, R. Collina and F. Delduc, Comm. Math. Phys. 120 (1988) 121.

[18] A. Hasenfratz and P. Hasenfratz, Nucl. Phys. B270 (1986) 687.

[19] M. Alford and J. March-Russell, Nucl. Phys. B417 (1993) 527. 